\title{
Uncultured Nitrospina-like species are major nitrite oxidizing bacteria in oxygen minimum zones
}

\author{
Xin Sun $\mathbb{D}^{1} \cdot$ Linnea F. M. Kop ${ }^{2} \cdot$ Maggie C. Y. Lau ${ }^{1,3} \cdot$ Jeroen Frank ${ }^{2} \cdot$ Amal Jayakumar $^{1} \cdot$ Sebastian Lücker $\mathbb{D}^{2} \cdot$ \\ Bess B. Ward $\left(^{1}\right.$
}

Received: 12 November 2018 / Revised: 6 May 2019 / Accepted: 9 May 2019 / Published online: 22 May 2019

(c) International Society for Microbial Ecology 2019

\begin{abstract}
Oxygen minimum zones (OMZs) are marine regions where $\mathrm{O}_{2}$ is undetectable at intermediate depths. Within OMZs, the oxygen-depleted zone (ODZ) induces anaerobic microbial processes that lead to fixed nitrogen loss via denitrification and anammox. Surprisingly, nitrite oxidation is also detected in ODZs, although all known marine nitrite oxidizers (mainly Nitrospina) are aerobes. We used metagenomic binning to construct metagenome-assembled genomes (MAGs) of nitrite oxidizers from OMZs. These MAGs represent two novel Nitrospina-like species, both of which differed from all known Nitrospina species, including cultured species and published MAGs. Relative abundances of different Nitrospina genotypes in OMZ and non-OMZ seawaters were estimated by mapping metagenomic reads to newly constructed MAGs and published high-quality genomes of members from the Nitrospinae phylum. The two novel species were present in all major OMZs and were more abundant inside ODZs, which is consistent with the detection of higher nitrite oxidation rates in ODZs than in oxic seawaters and suggests novel adaptations to anoxic environments. The detection of a large number of unclassified nitrite oxidoreductase genes in the dataset implies that the phylogenetic diversity of nitrite oxidizers is greater than previously thought.
\end{abstract}

\section{Introduction}

Fixed nitrogen in large areas of the ocean limits the growth of marine phytoplankton, which are responsible for half of the global photosynthetic carbon fixation [1,2]. Thus, fixed nitrogen is of vital importance to primary productivity and carbon cycling. Nitrate is the main nitrogen source for phytoplankton and is produced by nitrite oxidizing bacteria

Supplementary information The online version of this article (https:// doi.org/10.1038/s41396-019-0443-7) contains supplementary material, which is available to authorized users.

$\triangle$ Xin Sun

xins@princeton.edu

1 Department of Geosciences, Guyot Hall, Princeton University, Princeton, NJ 08544, USA

2 Department of Microbiology, Radboud University, Nijmegen, AJ 6525, the Netherlands

3 Present address: Laboratory of Extraterrestrial Ocean Systems, Institute of Deep-Sea Science and Engineering, Chinese Academic of Sciences, Sanya, Hainan 572000, China
(NOB). All known NOB are obligate aerobes, but Lipschultz et al. [3] and recent studies [4-7] reported the oxidation of nitrite to nitrate within oxygen minimum zones (OMZs). OMZs are characterized by a sharp $\mathrm{O}_{2}$ gradient (i.e., oxycline) overlying an oxygen depleted zone (ODZ) where the $\mathrm{O}_{2}$ concentration is low enough to induce anaerobic microbial processes, including denitrification and anaerobic ammonium oxidation (anammox). Since no anaerobic NOB have been identified, it remains unclear how nitrite oxidation occurs in this effectively anoxic environment.

Nitrite oxidation near the top of the ODZ may be fueled by nanomolar levels of $\mathrm{O}_{2}$ through episodic invasions of oxygenated seawater $[8,9]$ or by in situ production from photosynthesis in the deep chlorophyll maximum [10]. Nitrite oxidation in the dark core of the ODZ is more difficult to explain. Nitrite oxidation either occurred under nearly anoxic conditions (nanomolar oxygen levels; [11]) or was inhibited when oxygen increased to the micromolar range [5], implying the reaction was carried out by NOB specialists adapted to low $\mathrm{O}_{2}$ or anoxia.

Species affiliated with the phylum Nitrospinae are the dominant NOB in the marine environment [12-14]. The 
only two species in culture, Nitrospina gracilis and Nitrospina watsonii, were isolated under high (atmospheric level) $\mathrm{O}_{2}$ concentrations $[15,16]$. The genome of $N$. gracilis has been published [14], and the 16S ribosomal RNA (rRNA) gene sequence of $N$. watsonii is more than $97 \%$ identical to that of $N$. gracilis [15]. Their physiological characteristics suggest that these two Nitrospina species are unlikely to be responsible for apparently anaerobic nitrite oxidation in ODZs.

NOB diversity has been explored by sequencing Nitrospina-like 16S rRNA genes in seawater $[13,17]$ or nitrite oxidoreductase $(n x r)$ genes in marine sediments [18]. These approaches, however, do not allow linking the 16S rRNAinferred phylogeny to a functional gene (e.g., nxr)-derived physiological potential. Single cell genomics and metagenomic binning allow whole-genome reconstruction, which links taxonomic identities of uncultured species to their functional potentials. A recent study recovered novel Nitrospina-like single-cell amplified genomes (SAGs) from the ocean, but only two incomplete SAGs (13 and 21\% estimated genome completeness) originated from an OMZ [19].

In this study, we used metagenomics to investigate NOB in all three major OMZs, including the Eastern Tropical North (ETNP) and South Pacific (ETSP) and the Arabian Sea. We recovered metagenome-assembled genomes (MAGs) of two uncultured Nitrospina-like species (estimated completeness up to 93.1\%) from the ETNP and ETSP, which allowed us to analyze their genomic potentials and determine their relative abundance in all three major OMZs and global oxygenated seawaters.

\section{Materials and methods}

\section{Experimental site and sample collection}

Seawater samples were collected from a coastal station in the OMZ of the ETSP $\left(20.50^{\circ} \mathrm{S}, 70.70^{\circ} \mathrm{W}\right.$; Fig. S1) in 2013 on the R/V Nathaniel B. Palmer (NBP 1305). The vertical minimum $\mathrm{O}_{2}$ concentration was consistently below the STOX sensor detection limit (10 nM; [20]). Particulate material from $8 \mathrm{~L}$ of seawater was collected on $0.22-\mu \mathrm{m}$ Sterivex filters using a peristaltic pump. Sterivex filters were flash-frozen in liquid nitrogen onboard and stored at $-80^{\circ} \mathrm{C}$ until DNA extraction. Based on published profiles of $\mathrm{O}_{2}$, nutrients (Fig. S2; [21]) and nitrite oxidation rates [22], samples from four depths $(45,80,200$, and $300 \mathrm{~m})$ were selected for metagenomic sequencing.

\section{DNA extraction and sequencing}

DNA was extracted using the plant tissue protocol (All Prep DNA/RNA Mini Kit, Qiagen, Valencia, CA, USA) from
Sterivex filters. The quality of extracted DNA (length and concentration) was verified by the Agilent 2100 Bioanalyzer System. Randomly amplified DNA fragments were paired-end sequenced on an Illumina MiSeq to generate a total of 28 million read pairs (average length $350 \mathrm{bp}$ ) for four samples. DNA quality checking, random amplification, and sequencing were conducted by the Genomics Core Facility of Lewis-Sigler Institute for Integrative Genomics at Princeton University.

\section{Metagenomic analyses}

Raw reads were deposited on the Metagenomics Rapid Annotation using Subsystem Technology (MG-RAST) server [23] under the accession numbers mgm4842562.3, mgm4842563.3, mgm4842564.3, and mgm4842565.3. MG-RAST read annotation was performed to gain an overview of microbial community composition. The RefSeq database was chosen to classify all reads into different species based on best hits, and the Subsystem database to assign reads into different functional categories. $\alpha$-diversity of microbial species and functional genes was calculated based on classification results against the RefSeq database and the Subsystem database, respectively. Analyses targeting NOB are described below.

The four ETSP metagenomes from individual depths were combined, and the metagenome co-assembly and binning were performed using a pipeline developed inhouse. Briefly, the quality of the reads was assessed using FastQC (Babraham Bioinformatics, Babraham institute, Cambridge, UK). Reads were trimmed and quality-filtered using BBDuk from BBTools (DOE Joint Genome Institute, Walnut Creek, CA, USA) and assembled into metagenomic contigs using metaSPAdes [24]. Subsequently, the filtered reads were mapped to the assembled contigs using the Burrows-Wheeler Aligner [25] and SAMtools (v. 1.6, [26]) to obtain coverage information. Binning was performed using BinSanity [27], COCACOLA [28], CONCOCT [29], MaxBin 2.0 [30], and MetaBAT 2 [31]. Bins were dereplicated and aggregated using the DAS Tool [32], and their quality was assessed by checkM [33]. Two Nitrospina-like MAGs from ETSP metagenomes were submitted to NCBI under BioSample accession numbers SAMN10411459 (MAG-1) and SAMN10411419 (MAG-2). We also constructed one Nitrospina-like MAG from a published ETNP metagenome [34] using MetaBAT 2, but no Nitrospina-like MAG could be constructed from ETNP metagenomes from Glass et al. [35] or ETSP metagenomes from Stewart et al. [36].

Protein-coding sequences in the ETSP Nitrospina-like MAGs were predicted by Prodigal v.2.6.3 [37], and annotated using Prokka 1.12-beta [38] with the RefSeq bacterial $\mathrm{nr}$ protein database as a reference. Key genes (Table S3) 
were identified using a combination of the prokka annotation, best bi-directional BLAST hits with $N$. gracilis, and BLASTp searches. First, best bi-directional BLAST hits (evalue cutoff: $10^{-9}$ ) were determined between the amino acid sequences of $N$. gracilis and the two ETSP MAGs. Subsequently, the prokka annotation was searched for the remaining key genes. Presence or absence of genes in one or both MAGs was determined with BLASTp searches (evalue cutoff: $10^{-10}$ ). BLASTp hits with a bitscore higher than 59 were individually evaluated. No $n x r B$ genes were binned into either MAG, but we identified the best $n x r B$ candidates for the MAGs as follows: we assembled reads from each metagenome separately and got a MAG (MAG-80m) almost identical to ETSP MAG-1 (average nucleotide identity $=99.5 \%$ ), which contained a $n x r B$ sequence. We used this $n x r B$ sequence to search against all contigs from the co-assembly, and extracted the best hit (NODE_69234, 99.1\% identical). In addition, we calculated the corrected relative abundance of all $n x r B$ genes found in the ETSP metagenomes and compared them with the ETSP Nitrospina-like MAGs.

dRep [39] was used to select high-quality (completeness > $75 \%$ ), non-redundant genomes from all published genomes of members of the phylum Nitrospinae for further analyses. The selected genomes can be found in the NCBI database under the accession numbers GCA_000341545.2, GCA_002453875.1, GCA_001542995.1, GCA_001803525.1, GCA_002238965.1, GCA_002377645.1, GCA_002500605.1, GCA_002786505.1, GCA_002787235.1, GCA_002796165.1, and at IMG/M under the accession numbers 2681812871, 2651870060, 2651870063, and 2651870059. A phylogenetic tree of these genomes was constructed based on concatenated alignments of 92 core genes using the UBCG pipeline [40] with default settings. The genomes of the following four Nitrospira species served as outgroup: Nitrospira moscoviensis NSP M-1 (GCF_001273775.1), N. inopinata ENR4 (GCF_001458695.1), N. japonica NJ11 (GCF_900169565.1), and $N$. defluvii (GCF_000196815.1). The final tree was constructed from the concatenated UBCG alignment using RAxML v. 8.2.11 [41] with the "GTRCAT" protein model and 100 bootstraps replications.

The average nucleotide identity (ANI) between all available Nitrospinae genomes and the recovered Nitrospina-like MAGs was calculated using orthoANI [42]. Genome comparison among dereplicated published Nitrospinae genomes and newly constructed Nitrospina-like MAGs was performed with the anvi'o v.4 pangenomics workflow [43], using BLASTp to calculate similarities between amino acid sequences and with an MCL inflation parameter of 10 [44]. Unique genes in each OMZ MAG (Table S4A, B) and genes found in both OMZ MAGs, but not any of the other Nitrospinae genomes (Table S4C) were determined using the anvi'o interactive view of the pangenome. Amino acid sequences were annotated using BLASTp searches (e-value cutoff: $10^{-10}$ ) against the Swissprot and the NCBI nr databases. Hits shorter than 100 bp and eukaryotic hits were removed.

Contigs in Nitrospina-like MAGs were searched against the SILVA-119 database using BLASTn in order to identify Nitrospina-like rRNA genes (ETSP MAG-1: 427 bp, ETSP MAG-2: $882 \mathrm{bp}$ ). A 16S rRNA gene reference set was constructed by blasting (BLASTn) the $N$. gracilis $16 \mathrm{~S}$ rRNA gene sequence (NR_104821.1) against the NCBI nt database. The 16S rRNA gene sequences of the top 250 hits were then clustered with usearch [45] using an identity threshold of 0.99 . In addition, 16S rRNA sequences from published Nitrospinae genomes were extracted by blasting the genome sequence against the SILVA-132 database. All 16S rRNA gene sequences were aligned using MUSCLE v. 3.8.425 (alignment length $1644 \mathrm{bp}$; [46]) and a phylogenetic tree was calculated without use of conservation filters in RAxML v. 8.2.11 [41] with the "GTRCAT" protein model and 100 bootstraps replications.

$n x r B$ genes in the metagenome were identified by blasting (tBLASTn) the $N$. gracilis $n x r B$ sequence (AGF29467.1) against all contigs. Only hits with identity $>50 \%$ to the $N$. gracilis $n x r B$ and a minimum length of 390 bp were used for the subsequent analysis. The $n x r B$ tree was constructed from a MUSCLE alignment (v. 3.8.425, alignment length $512 \mathrm{bp}$; [46]) without use of conservation filters in RAxML v. 8.2.11 [41] with the rapid bootstrapping algorithm (100 boostraps) using automatic protein model selection (PROTGAMMAAUTO). Selenate reductase sequences served as outgroup. Trees were visualized in iTOL v4.2.3 [47].

The occurrence of the two newly constructed Nitrospinalike MAGs in all three major OMZs was determined by mapping reads from this study and published metagenomic data from the ETNP [35], the ETSP [36] and Arabian Sea [48] to the MAGs using Bowtie2 (v. 2.3.1; [49]) and SAMtools (v. 1.6; [26]). In order to determine the presence of the OMZ Nitrospina-like MAGs in the surface ocean, 12 co-assemblies of TARA Oceans metagenomes [50] were screened. To limit the use of computational resources, unique genes of the MAGs determined with the anvi'o pangenomics workflow were blasted (tBLASTn) against the co-assemblies first. If there were hits with more than $98 \%$ identity for a certain co-assembly, unique genes were blasted (tBLASTn) against all individual metagenomes generating this co-assembly. Subsequently, read mapping was performed for those individual metagenomes that had hits with more than $98 \%$ identity. The presence of other Nitrospinae genomes in the ETSP OMZ was determined by mapping metagenomic reads from the ETSP OMZ to these genomes as described above. All Bowtie2 mappings were conducted without trimming ("end-to-end") and using the 
"very-sensitive" preset mode. Only reads with a mapping quality above 20 were included. Relative abundances were calculated by dividing the number of reads mapped to the MAG by the total number of reads in the sample.

\section{Results and discussion}

\section{Microbial community composition varies with depth in the ETSP OMZ}

The presence of an ODZ at the sampling station in ETSP was indicated by the local nitrite maximum, nitrate deficit and undetectable $\mathrm{O}_{2}$ concentration at $75-400 \mathrm{~m}$ (Fig. S2). The $45 \mathrm{~m}$ sample was from oxic surface seawater $\left(\left[\mathrm{O}_{2}\right]=\right.$ $\left.85 \mu \mathrm{M},\left[\mathrm{NO}_{2}{ }^{-}\right]=0.3 \mu \mathrm{M}\right)$, the $80 \mathrm{~m}$ sample was from the top of the ODZ $\left(\left[\mathrm{O}_{2}\right]<10 \mathrm{nM},\left[\mathrm{NO}_{2}{ }^{-}\right]=0.3 \mu \mathrm{M}\right)$, and both $200 \mathrm{~m}$ and $300 \mathrm{~m}$ samples were from the anoxic core of the ODZ (undetectable $\mathrm{O}_{2},\left[\mathrm{NO}_{2}{ }^{-}\right]>6 \mu \mathrm{M}$ ).

Variations in chemical and physical conditions with depth in the ETSP OMZ corresponded to a shift in microbial communities. Microbial species $\alpha$-diversity was highest at the top of the ODZ ( $80 \mathrm{~m}$; Fig. S3). The $\alpha$-diversity at 45 $\mathrm{m}$ was similar to those at $200 \mathrm{~m}$ and $300 \mathrm{~m}$, but the microbial communities in oxic and anoxic environments differed in composition (Fig. S4). For example, Proteobacteria (about half of which are Pelagibacter spp.) were relatively more abundant in samples from the core of the ODZ (200 m, $300 \mathrm{~m})$. The prevalence of Pelagibacter spp. in the anoxic ODZ core is consistent with the nitrate reducing capacity of some Pelagibacter clades, previously reported in the ETNP [34]. Thaumarchaeota (ammoniaoxidizing archaea, AOA) had high relative abundances at $45 \mathrm{~m}$ and $80 \mathrm{~m}$, but were undetectable in the core of the ODZ. Consistently, the AOA Nitrosopumilus maritimus was identified as one of the most abundant species only in the surface of the ETSP OMZ [36]. This observed confinement of AOA to oxygenated water layers was likely driven by $\mathrm{O}_{2}$ availability, since ammonia oxidation in AOA is an obligate aerobic process.

The shift in microbial communities was also observed in functional gene-based analyses, where the $\alpha$-diversity decreased with increasing depth (Fig. S3). One example of functional gene variation among communities from different depths was the absence of photosynthesis genes in the dark core of the ODZ (Fig. S5).

\section{Two novel Nitrospina-like species were discovered in ODZs}

Four Nitrospina-like MAGs were constructed from the new ETSP metagenomes and one MAG from a previously published ETNP metagenome ([34]; Table S1). Based on single-copy gene counts [33], the ETSP MAG-1 and MAG2 both had an estimated completeness of $93.1 \%$, and the ETNP MAG of 77.1\%. Due to the low completion of ETSP MAG-3 and MAG-4 (completeness $<30 \%$ ), these were excluded from later analyses. The fact that multiple Nitrospina-like MAGs were recovered, however, suggests that distinct Nitrospina-like NOB populations co-exist within OMZs. The GC content $(45.6 \%$ for ETSP MAGs and $45.7 \%$ for the ETNP MAG, Table S1) of OMZ MAGs was consistently lower than that of Candidatus Nitromaritima RS ( 50.0\%; [51]), N. gracilis (57.3\%; [14]), and $N$. watsonii $(55.6 \%$; [15]), but similar to that of a Nitrospinalike MAG recovered from the Northern Gulf of Mexico (45.5\%; [52]).

Based on the established genus and species cutoff thresholds (ANI $<70 \%$ and $<95 \%$, respectively; [53]), pairwise genome comparisons indicated that the three MAGs represent two novel species without cultured representatives (Fig. 1). Although from two different geographic regions, ETSP MAG-1 and ETNP MAG were highly similar to each other $(\mathrm{ANI}=99.6 \%)$ and likely represent the same species, which is distinct from ETSP MAG-2 $(\mathrm{ANI}=83.6 \%$; Fig. 1$)$. Due to this high genomic identity and the higher completeness of ETSP MAG-1 based on the dRep analysis, ETNP MAG was excluded from later analysis. Both remaining MAGs differed from all publicly available Nitrospina-like species at the species level (ANI < $75 \%$ ) and from $N$. gracilis (the only cultured representative for which a genome is available) at the genus level (ANI < $67 \%$ ). The novelty of the OMZ MAGs was also confirmed by phylogenetic analyses based on concatenated bacterial core protein alignments encompassing a dereplicated subset of all published high-quality (genome completeness $>75 \%$ ) Nitrospina-like genome representatives (Fig. 1). In the resulting tree, the two ETSP MAGs formed a separate clade with two draft genomes from the North Atlantic Gyre (Nitrospina sp. AG-538-K21 and AB-629-B18) as closest relatives. This phylogenetic divergence of the two ETSP MAGs was also indicated by 16S rRNA gene-based phylogenetic analyses (Fig. 2). In the tree containing 16S rRNA gene sequences of the two ETSP MAGs, Nitrospina 16S rRNA gene sequences, and long sequences (>1000 bp) from all publicly available Nitrospina-like genomes, sequences of ETSP MAGs are closest to sequences recovered from the northeast subarctic Pacific Ocean OMZ, indicating that Nitrospina-like organisms found in OMZs are phylogenetically distinct from other marine NOB, including the two cultured representatives, $N$. gracilis and $N$. watsonii. This genome-level divergence implies greater functional diversity among NOB, as previously suggested [54].

The observed differences of the novel Nitrospina-like OMZ MAGs from cultured representatives and all MAGs derived from non-OMZ environments indicate niche 

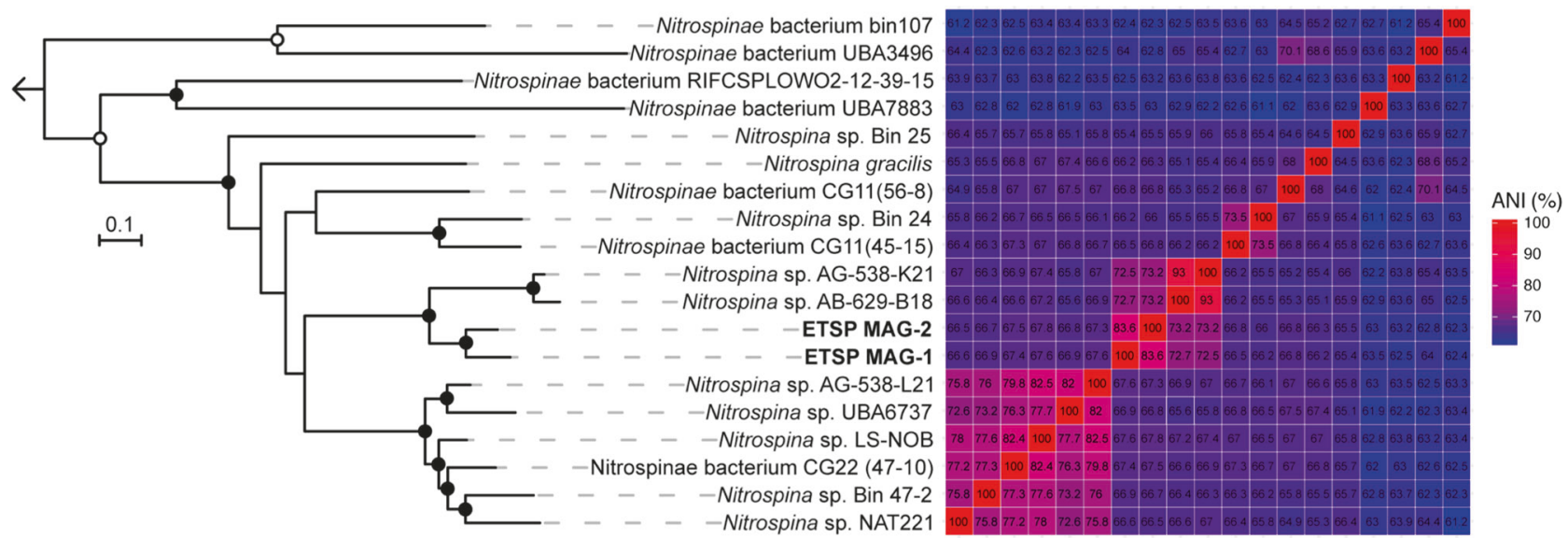

Fig. 1 Phylogenetic placement of the two Nitrospina-like MAGs from the ETSP OMZ metagenomes (in bold) based on concatenated alignments of 92 core protein sequences. The tree contains all available non-redundant Nitrospinae genomes with an estimated completeness $>75 \%$. Open and closed circles represent bootstrap support $\geq 90 \%$ and

differentiation of Nitrospina species in the ocean. $\mathrm{O}_{2}$ and $\mathrm{NO}_{2}{ }^{-}$might be the primary drivers of this differentiation, since non-OMZ marine environments usually have much higher $\mathrm{O}_{2}$ but much lower $\mathrm{NO}_{2}{ }^{-}$concentrations. In addition, $\mathrm{O}_{2}$ and $\mathrm{NO}_{2}{ }^{-}$concentrations used to enrich $N$. gracilis [16] were at least 100-fold higher than those found in the OMZ.

\section{OMZ NOB are more diverse than previously thought}

Functional genes often are conserved across phylogenetically distant species. Since the organisms responsible for nitrite oxidation in OMZs remain unknown, using the $n x r$ gene as a proxy for nitrite oxidizing capacity may identify novel microorganisms mediating this reaction. We used the $N$. gracilis $n x r B$ gene sequence to probe for related genes in the metagenomic assemblies. We also estimated their relative abundance in the ETSP OMZ (Fig. S6). None of the 17 $n x r B$-like genes present in the metagenome could unequivocally be linked to either of the two MAGs, but based on consistent relative abundance distribution of genes versus MAGs (Fig. S6) and the association of a $n x r B$ gene in the

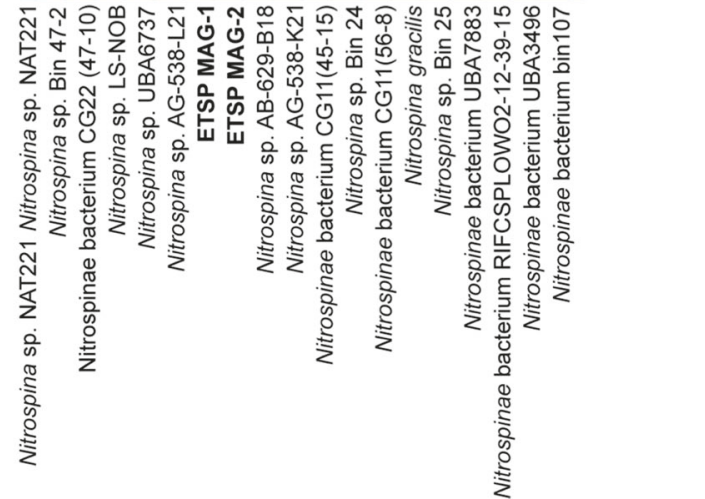

$=100 \%$, respectively. The arrow indicates the location of the outgroup (Nitrospira). The scale bar represents $10 \%$ estimated sequence divergence. In the right panel, the average nucleotide identities $(\%)$ between the Nitrospinae genomes are shown

ETSP MAG-80m, we have identified likely candidates. We propose that NODE_69234 and NODE_114897 contain the best $n x r B$ candidates for MAG-1 and MAG-2, respectively. Both of these two $n x r B$ genes and another $n x r B$ gene were highly similar to the $n x r B$ of $N$. gracilis (NODE_102582, 96.9\%; NODE_69234, 96.7\%; and NODE_114897, 96.7\% identity) and clustered with Nitrospina $n x r B$ genes in the tree (Fig. 3). This analysis implies that the two novel OMZ MAGs represent Nitrospina-like species mediating nitrite oxidation in the ETSP OMZ. Two other $n x r B$-like sequences (NODE_1303 and NODE_9134) cluster together with anammox (Fig. 3), which is consistent with the detection of anammox activity at the same time in this OMZ [22] and the ability of anammox bacteria to oxidize nitrite to nitrate.

Moreover, a group of $n x r B$-like sequences formed a novel sequence cluster between the anammox and Nitrospina $n x r B$ clusters. This group is most closely related to MAGs recovered from a suboxic/anoxic aquifer near Rifle $(\mathrm{CO}$, USA; [55]). It is tempting to speculate that these putative $n x r B$ sequences represent novel, yet unidentified nitrite oxidizers, as was also implied by a recent gene-centric nxrAbased analysis performed in the Arabian Sea [48]. 


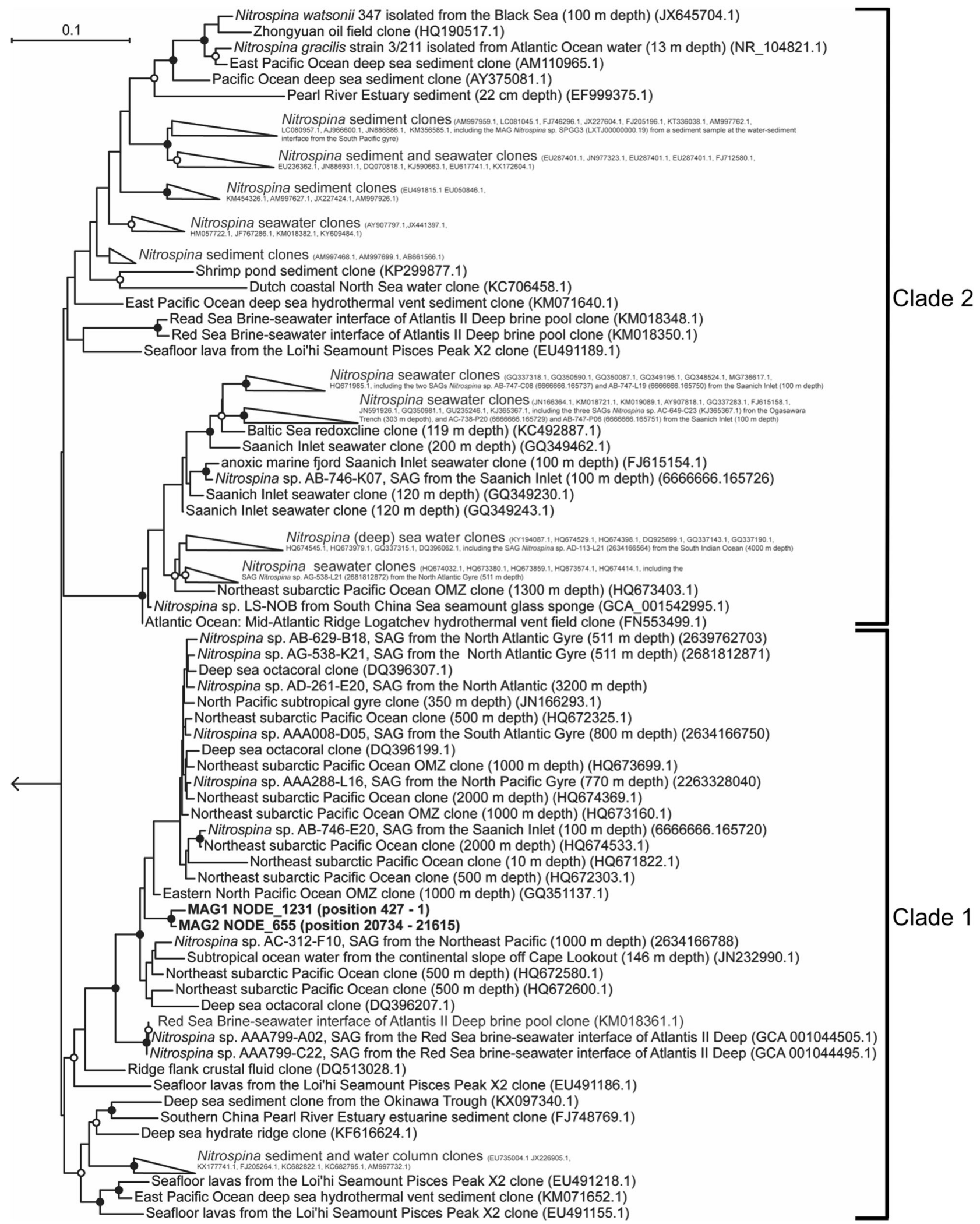

Fig. 2 16S rRNA gene-based phylogenetic analysis of selected members of the genus Nitrospina. Sequences of the two ETSP OMZ MAGs from this study are indicated in bold. Open and closed circles represent bootstrap values $\geq 70 \%$ and $\geq 90 \%$, respectively. The arrow indicates the position of the outgroup (Nitrospira). The scale bar corresponds to $10 \%$ estimated sequence divergence. The tree was calculated using the rapid bootstrapping algorithm (100 bootstrap iterations). No conservation filter or weighting mask was used, resulting in 1644 valid alignment positions 


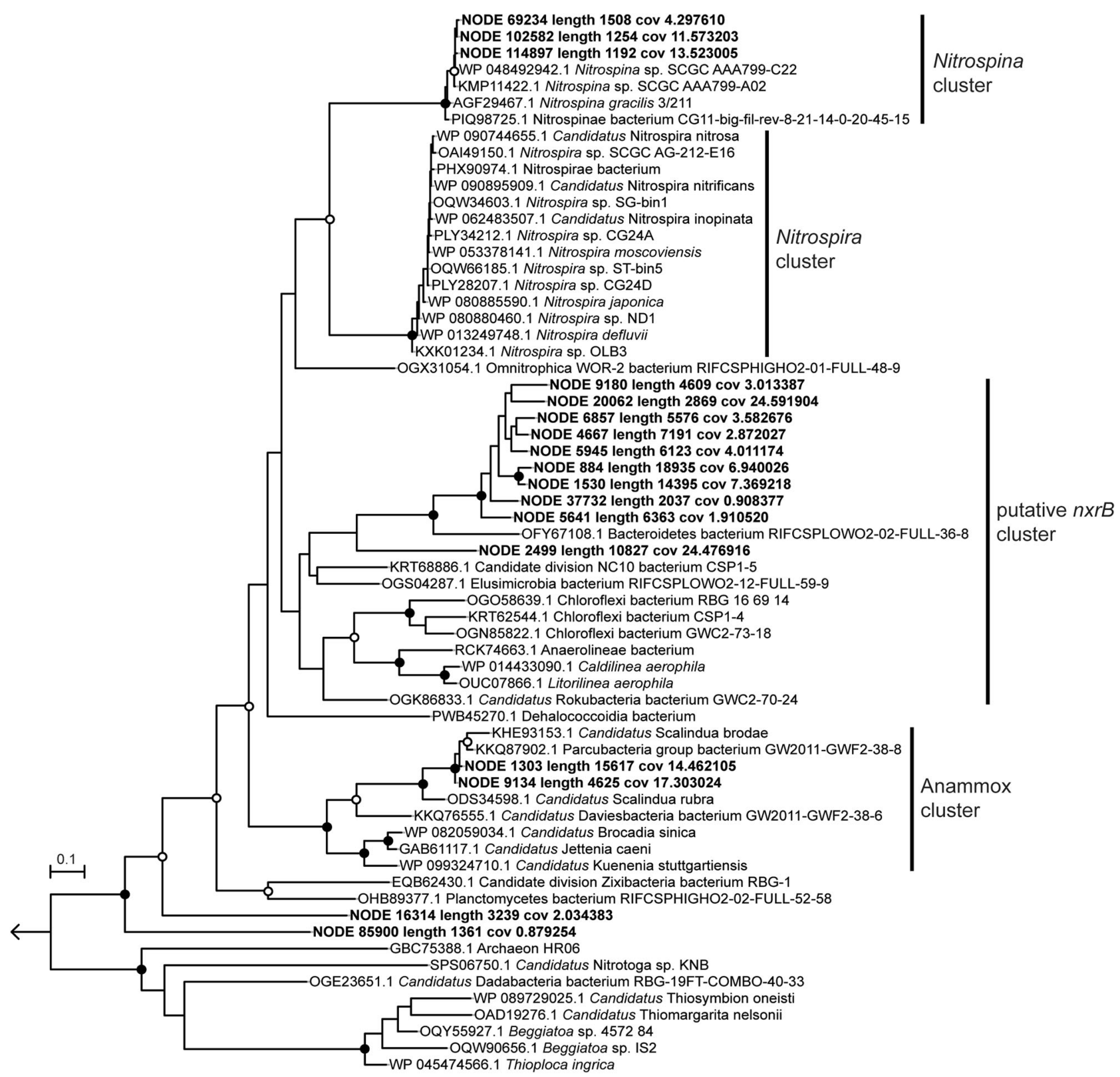

Fig. 3 Phylogenetic analysis of selected $n x r B$-like sequences. Sequences from the ETSP OMZ metagenomes are indicated in bold. Open and closed circles represent bootstrap values $\geq 70 \%$ and $\geq 90 \%$, respectively. The arrow indicates the position of the outgroup (selenate

Lastly, there are two $n x r B$ sequences (NODE_16314 and NODE_85900) only distantly related to classified $n x r B$ genes. Their low identities to sequences represented in the NCBI nr database (NODE_16314, 55\% identity to $n x r B$ from Candidatus Scalindua brodae [KHE93153.1]; NODE_85900, 57\% identity to hypothetical protein RBG1_1C00001G0009 from the candidate division Zixibacteria bacterium RBG-1 [EQB62430.1]), their distinct clustering in the phylogenetic tree (Fig. 3), and the fact that they could not be binned into any MAG leave their exact function as well as their phylogenetic affiliation unclear. reductase sequences). The scale bar corresponds to $10 \%$ estimated sequence divergence. The tree was calculated with a rapid bootstrapping algorithm (100 bootstrap iterations). No conservation filter was used, resulting in 512 alignment positions

However, a role in either nitrite oxidation or nitrate reduction is highly probable.

\section{Distribution of Nitrospina-like MAGs and implications for nitrite oxidation in OMZs}

The relative abundances of ETSP MAG-1 and MAG-2 were determined in order to estimate their distributions in three major OMZs (Fig. 4). Both species were very rare in oxygenated surface seawaters. MAG-1 was more abundant than MAG-2 at the top of the ODZ, but the relative abundance of 


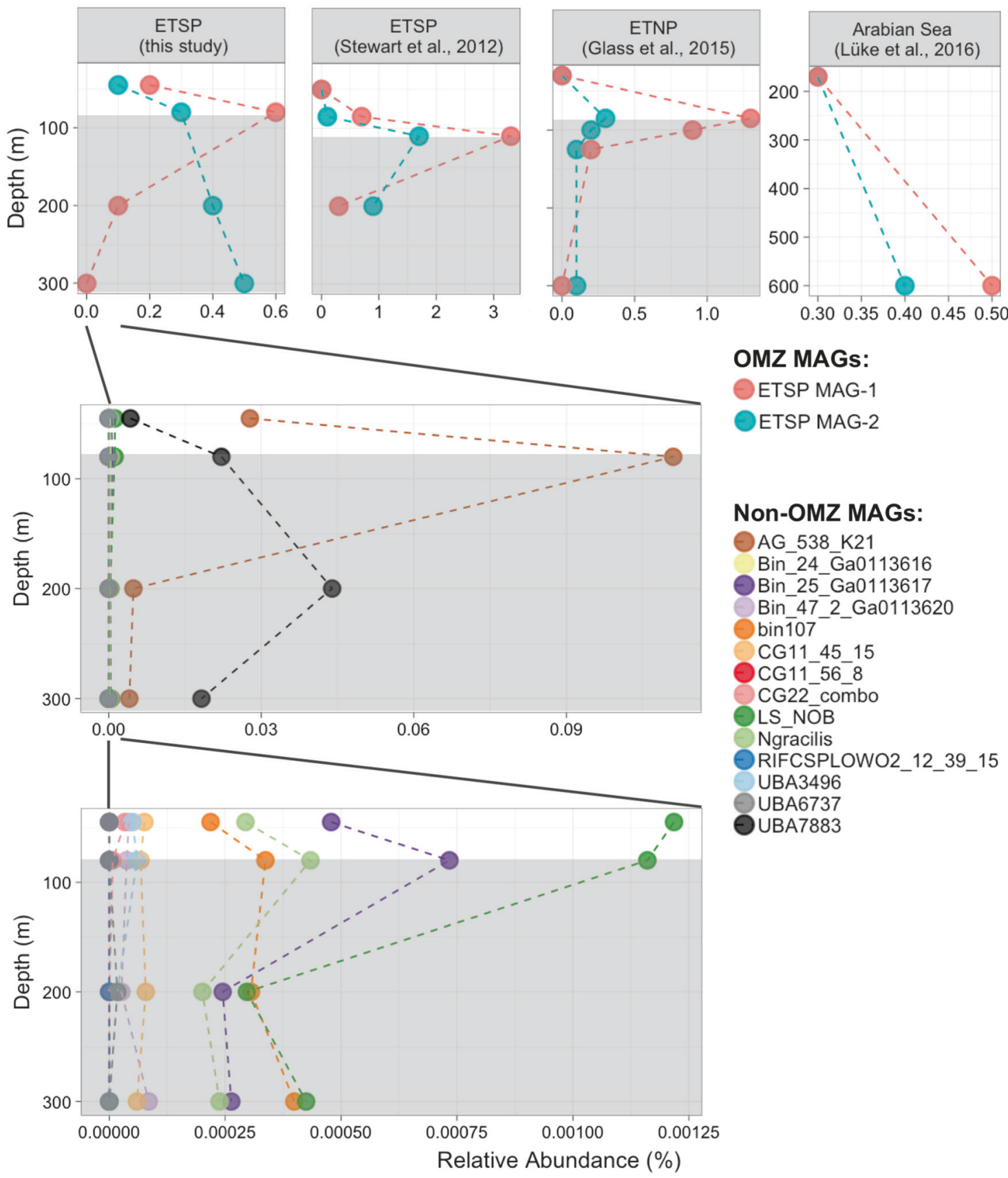

Fig. 4 Relative abundance of OMZ MAG-1 and MAG-2 in ETSP, ETNP, and Arabian Sea OMZ metagenomes from this and other studies, and relative abundance of non-OMZ Nitrospina-like MAGs in ETSP metagenomes from this study. Shaded areas in the plots indicate the location of the anoxic ODZ. Dashed lines between dots are used as a visual guide to differentiate different $\mathrm{NOB}$, they do not indicate linear changes

was slightly above detection limit $(2.5 \mu \mathrm{M})$, and the $\mathrm{NO}_{2}{ }^{-}$ concentration was only $0.5 \mu \mathrm{M}$ [56]. Conditions at $600 \mathrm{~m}$ of the Arabian Sea are more similar to the environments at the top of the ODZ in the ETSP and ETNP than to the ODZ core, where $\mathrm{O}_{2}$ is below $10 \mathrm{nM}$ and $\mathrm{NO}_{2}{ }^{-}$is at $\mu \mathrm{M}$ levels. These contrasting distributions imply different $\mathrm{O}_{2}$ preferences, although both MAGs are present at all depths. Conditions at the top of the ODZ apparently allow the 
coexistence of different NOB adapted to either low $\mathrm{O}_{2}(\mathrm{nM}$ level) or anoxic conditions. Nitrite oxidation rates in OMZs either increased with only $\mathrm{nM}$ levels of $\mathrm{O}_{2}$ addition [11] or decreased when $\mathrm{O}_{2}$ addition reached $\mu \mathrm{M}$ levels [5, 57]. One possible explanation for this paradox is that samples from different sites were dominated by NOB adapted to different $\mathrm{O}_{2}$ conditions.

The relative abundance of Nitrococcus mobilis is comparable with Nitrospina-like MAGs only in the Arabian Sea OMZ $(0.27 \%$ at $170 \mathrm{~m}$ and $0.43 \%$ at $600 \mathrm{~m})$. In all other OMZs (ETNP, ETSP-Stewart, ETSP-this study), Nitrococcus was not detected. The other Nitrospinae species could only be found at very low abundances in this dataset of the ETSP OMZ (Fig. 4). In addition, OMZ Nitrospinalike species are the only NOB species detected by binning from ETSP and ETNP metagenomes. These results suggest that OMZ Nitrospina-like NOB, rather than Nitrococcus or other Nitrospina species (i.e., $\mathrm{N}$. gracilis or $\mathrm{Ca}$. Nitromaritima RS), are the dominant NOB in the OMZ. At the same time that our metagenomic samples were collected, Babbin et al. [22] detected nitrite oxidation in incubation bottles in which both anaerobic nitrogen-loss processes, anammox and denitrification, were also detected. The distribution of nitrite oxidation rates with depth was consistent with that of the estimated relative abundance of the two MAGs recovered here (Fig. 4), suggesting these novel species were indeed responsible for the measured rates.

Contrastingly, in non-OMZ metagenomes from the surface ocean, the OMZ Nitrospina-like MAGs were not found, or were detected only at very low relative abundances (Table S2). Together with the phylogenetic distance between OMZ and non-OMZ NOB (Figs. 1 and 2), these results indicate that the Nitrospina-like MAGs found in this study are unique to OMZ environments. The detection of novel unclassified $n x r B$ genes (Fig. 3 and Fig. S6), however, indicates that other species may also contribute to nitrite oxidation in OMZs. Since it is usually easier to recover (draft) genomes from more abundant species, other less abundant species may have been captured only partly, and the total relative abundance of NOB in OMZs thus was likely underestimated by our approach.

\section{Metabolic pathways of OMZ MAGs}

Although neither of the two Nitrospina-like MAGs is $100 \%$ complete, it is useful to evaluate presence and absence of metabolic pathways to assess the biogeochemical role and metabolic potential of the new organisms, and to motivate further research on key pathways. Some of the genes identified as "missing" here might be present in the complete genome, and thus targeted efforts should be made to verify their absence. Adaptations in their respiratory pathways might allow the OMZ Nitrospina-like species to occupy low $\mathrm{O}_{2}$ or anoxic niches. ETSP MAG-1 encodes a b (a/o)3-type (type B) cytochrome $c$ oxidase, which could function as terminal oxidase in this organism (Tables S3 and S4). This contrasts with $N$. gracilis, which encodes a $c b b_{3}$-type terminal oxidase (C-type). Both B- and C-type oxidases have higher affinities than those belonging to type A, which indicates that Nitrospina generally are well adapted to low-oxygen environments [58]. Thus, NOB from ODZs with high-affinity terminal oxidases might be able to start oxidizing nitrite when trace amounts of $\mathrm{O}_{2}$ are supplied. These NOB might be the major contributors to the nitrite oxidation rates measured [5] and modeled [8] at both upper and lower boundaries, where $\mathrm{O}_{2}$ diffuses into the ODZ. However, a cytochrome $c$ oxidase could not be found in ETSP MAG-2 (Tables S3 and S4). If this lack of high $\mathrm{O}_{2}$ affinity terminal oxidases in MAG-2 is not due to the incompleteness of the draft genome, it could explain why MAG-2 is less abundant than MAG-1 at the ODZ top where nM levels of $\mathrm{O}_{2}$ are available (Fig. 4). Moreover, this might indicate the presence of novel unidentified cytochrome $c$ oxidases, or the use of alternative pathways to oxidize nitrite anaerobically. Similar to N. gracilis, both MAGs encode proteins with limited similarity to cytochrome $b d$ type quinol oxidases, which are also conserved in all genome-sequenced Nitrospira and anammox species [59]. However, based on the lack of quinol-binding sites, they are unlikely to function as canonical cytochrome $b d$ oxidases [14], and the possible role of these cytochrome bd-like oxidases in nitrite oxidation remains unknown.

Most genes involved in oxidative phosphorylation are present in both ETSP MAGs (Table S3). Consistent with known Nitrospina species [14, 19], the novel Nitrospinalike NOB fix $\mathrm{CO}_{2}$ via the reductive tricarboxylic acid cycle (rTCA; Table S3). While two of the three key genes for the rTCA cycle, ATP-dependent citrate lyase (ACL), and 2oxoglutarate:ferredoxin oxidoreductase (OGOR) were detected in both MAGs, not all subunits of the pyruvate: ferredoxin oxidoreductase (POR) were identified. The apparent absence of two of the subunits of POR is likely due to genome incompleteness and insufficient sequencing depth, as some subunits of the complex were found (Table S3). In addition, there was no indication of other $\mathrm{CO}_{2}$ fixation pathways in these Nitrospina-like organisms, such as Calvin-Benson-Bassham cycle used by Nitrococcus mobilis [57]. The use of the rTCA cycle by the novel Nitrospina-like species is consistent with their apparent preference for low energy, low-oxygen environments [14, 19].

Although $\mathrm{O}_{2}$ diffusion could influence waters just below the oxycline, nitrite oxidation occurs in the core of the ODZ where such $\mathrm{O}_{2}$ supply is not possible. While OMZ NOB were speculated to couple nitrite oxidation to iodate reduction [22], this is unlikely to be the explanation of 
apparently anaerobic nitrite oxidation NOB in ODZs because iodate, similar to $\mathrm{O}_{2}$, is also depleted in ODZ cores [60]. Anaerobic nitrite oxidation can be catalyzed by anoxygenic photosynthesis [61], which, however, is also unlikely in the dark ODZ core. It should be noted that although anammox bacteria also oxidize nitrite anaerobically to nitrate, the low anammox rates at this site could not explain the measured nitrite oxidation rates. One possible explanation for this observation is the production of nitrate through nitrite dismutation [62]. To date, only one study directly explored the possibility for nitrite dismutation in the Namibian OMZ, but failed to detect this process [57]. However, further studies in other OMZs are needed to test this possibility. Anaerobic nitrite oxidation may be mediated by novel Nitrospina-like species discovered in this study, or by other yet unidentified species as indicated by the detection of unclassified putative $n x r B$ genes (Fig. 3). One possibility we cannot rule out is that the in situ nitrite oxidation rates were zero, especially at the ODZ core, and that measured nitrite oxidation rates were fueled by trace $\mathrm{O}_{2}$ contamination during sampling. The detection of Nitrospina-like species by both marker gene analyses and metagenomic binning, however, implies that these bacteria have the potential to oxidize nitrite with terminal electron acceptors other than $\mathrm{O}_{2}$, or conserve energy by alternative anaerobic pathways for survival in anoxic environments. The potential of the two MAGs to reduce nitrite is indicated by the presence of nitrite reductase genes, but no nitric oxide reductase gene or nitrous oxide reductase gene was recognized in the MAGs, as is the case for all NOB genomes. Therefore, it is unlikely that the OMZ NOB play a role in complete denitrification. The possibility of conducting nitrate respiration when conditions do not favor nitrite oxidation has been shown for the NOB Nitrococcus [57] and Nitrospira [63]. Thus, OMZ NOB might survive in ODZs by alternative anaerobic metabolisms and turn on the conventional nitrite oxidation pathway when minute amounts of $\mathrm{O}_{2}$ become available.

Although perhaps not central to the nitrite oxidation metabolism, the draft genomes contained additional features that provide insights into the ecology of the OMZ NOB. In contrast to $N$. gracilis [14], the two ETSP MAGs do not encode a sulfhydrogenase or a sulfite:cytochrome $c$ oxidoreductase, making it unlikely that these organisms use sulfur compounds for energy conservation. It is known that some nitrifiers can use urea or cyanate as nitrogen source for assimilation or as indirect energy source in a reciprocal feeding mechanism in symbiosis with ammonia-oxidizing microorganisms (AOM; [63, 64]). While neither of the ETSP MAGs possesses a cynS gene, the presence of genes encoding a urease and urea transporters in both MAGs indicates their potential to use urea. In reciprocal feeding, they would provide ammonia from urea degradation to the
$\mathrm{AOM}$ and would receive their energy source nitrite in return. Chlorite dismutase precursor genes were found in both ETSP MAGs. Since the Nxr can catalyze chlorate reduction to chlorite [65], these novel species have the potential to reduce chlorate. Chlorate is not abundant in anoxic marine environments, so the chlorite dismutase genes might support some unidentified alternative anaerobic pathway. Lastly, both OMZ MAGs encoded a siderophore transport systems, but neither of them has a siderophore biosynthesis pathway (Table S3). Many microbes produce siderophores to scavenge essential metals, mainly iron, from the environment [66]. The presence of siderophore transporters indicates that these Nitrospina-like organisms may take advantage of siderophores produced by other organisms in the OMZ, as has been suggested for the ammoniaoxidizing bacterium Nitrosomonas europaea [67].

\section{Conclusions}

Three novel Nitrospina-like MAGs from ETSP and ETNP OMZs, representing two novel NOB species, were reconstructed from newly obtained and previously published metagenomic data. They represent nearly complete genomes of two novel species, which lack cultured representatives and are distinct from $N$. gracilis and $N$. watsonii at the genus level. The relative abundance patterns of these novel NOB were consistent with the distribution of nitrite oxidation rates in OMZ environments. The OMZ MAGs were rarely observed in metagenomic datasets from oxygenated surface seawaters, and the NOB recovered from oxygenated regions are rarely present in OMZs, implying niche partitioning of marine NOB species. These novel Nitrospina-like OMZ NOB, along with additional unidentified putative nitrite oxidizers indicated by the presence of unclassified $n x r B$-like sequences, may contribute to the apparently anaerobic nitrite oxidation detected in OMZs, or may use alternative pathways to survive in ODZs when conditions are not favorable for nitrite oxidation. The discovery of novel NOB species in an environment thought to be unsuitable for NOB suggests that the versatility and diversity of NOB thus may be even larger than previously recognized.

Acknowledgements This study was funded by NSF grants to BBW and AJ. LFMK, JF, and SL were supported by the Netherlands Organization for Scientific Research (grants 863.14.019, 016. Vidi.189.050, and SIAM Gravitation Grant 024.002.002). We would like to acknowledge all scientists and the crew of the R/V Nathaniel B. Palmer for assistance in sample collection. We are grateful to Wei Wang for his help in the Princeton Genomics Core Facility. We also thank two anonymous reviewers for their very helpful insights.

Author contributions XS and BBW planned the study. XS and LFMK performed all metagenomic analyses. JF and MCYL set up pipelines 
for metagenomic analyses. AJ provided DNA samples. XS, LFMK, $\mathrm{SL}$, and BBW analyzed data and wrote the paper. All authors approved the final paper.

\section{Compliance with ethical standards}

Conflict of interest The authors declare that they have no conflict of interest.

Publisher's note: Springer Nature remains neutral with regard to jurisdictional claims in published maps and institutional affiliations.

\section{References}

1. Arrigo KR. Marine microorganisms and global nutrient cycles. Nature. 2005;437:343-8.

2. Tyrrell T. The relative influences of nitrogen and phosohorus on oceanic primary production. Nature. 1999;400:525-31.

3. Lipschultz F, Wofsy SC, Ward BB, Codispoti LA, Friedrich G, Elkins JW. Bacterial transformations of inorganic nitrogen in the oxygen-deficient waters of the Eastern Tropical South Pacific Ocean. Deep Sea Res Part A, Oceanogr Res Pap. 1990;37:1513-41.

4. Füssel J, Lam P, Lavik G, Jensen MM, Holtappels M, Gunter M, et al. Nitrite oxidation in the Namibian oxygen minimum zone. ISME J. 2012;6:1200-9.

5. Sun X, Ji Q, Jayakumar A, Ward BB. Dependence of nitrite oxidation on nitrite and oxygen in low oxygen seawater. Geophys Res Lett. 2017;44:7883-91.

6. Beman JM, Shih JL, Popp BN. Nitrite oxidation in the upper water column and oxygen minimum zone of the eastern tropical North Pacific Ocean. ISME J. 2013;7:2192-205.

7. Casciotti KL, Buchwald C, McIlvin M. Implications of nitrate and nitrite isotopic measurements for the mechanisms of nitrogen cycling in the Peru oxygen deficient zone. Deep Res Part I Oceanogr Res Pap. 2013;80:78-93.

8. Peters BD, Babbin AR, Lettmann KA, Mordy CW, Ulloa O, Ward $\mathrm{BB}$, et al. Vertical modeling of the nitrogen cycle in the eastern tropical South Pacific oxygen deficient zone using high-resolution concentration and isotope measurements. Global Biogeochem Cycles. 2016;30:1661-81.

9. Thamdrup B, Dalsgaard T, Revsbech NP. Widespread functional anoxia in the oxygen minimum zone of the Eastern South Pacific. Deep Res Part I Oceanogr Res Pap. 2012;65:36-45.

10. Garcia-Robledo E, Padilla CC, Aldunate M, Stewart FJ, Ulloa O, Paulmier A, et al. Cryptic oxygen cycling in anoxic marine zones. Proc Natl Acad Sci. 2017;114:201619844.

11. Bristow LA, Dalsgaard T, Tiano L, Mills DB, Bertagnolli AD, Wright JJ, et al. Ammonium and nitrite oxidation at nanomolar oxygen concentrations in oxygen minimum zone waters. Proc Natl Acad Sci. 2016;113:10601-6.

12. Ganesh S, Bristow LA, Larsen M, Sarode N, Thamdrup B, Stewart FJ. Size-fraction partitioning of community gene transcription and nitrogen metabolism in a marine oxygen minimum zone. ISME J. 2015;9:2682-96.

13. Levipan HA, Molina V, Fernandez C. Nitrospina-like bacteria are the main drivers of nitrite oxidation in the seasonal upwelling area of the Eastern South Pacific (Central Chile $\sim 36^{\circ} \mathrm{S}$ ). Environ Microbiol Rep. 2014;6:565-73.

14. Lücker S, Nowka B, Rattei T, Spieck E, Daims H. The genome of Nitrospina gracilis illuminates the metabolism and evolution of the major marine nitrite oxidizer. Front Microbiol. 2013;4:1-19.

15. Spieck E, Keuter S, Wenzel T, Bock E, Ludwig W. Characterization of a new marine nitrite oxidizing bacterium, Nitrospina watsonii sp. nov., a member of the newly proposed phylum "Nitrospinae.". Systermatic Appl Microbiol. 2014;37:170-6.

16. Watson SW, Waterbury JB. Characteristics of two marine nitrite oxidizing bacteria, Nitrospina gracilis nov. gen. nov. sp. and Nitrococcus mobilis nov. gen. nov. sp. Microscopy. 1971;77:203-30.

17. Mincer TJ, Church MJ, Taylor LT, Preston C, Karl DM, DeLong EF. Quantitative distribution of presumptive archaeal and bacterial nitrifiers in Monterey Bay and the North Pacific Subtropical Gyre. Environ Microbiol. 2007;9:1162-75.

18. Rani S, Koh HW, Rhee SK, Fujitani H, Park SJ. Detection and diversity of the nitrite oxidoreductase alpha subunit (nxrA) gene of nitrospina in marine sediments. Microb Ecol. 2017;73:111-22.

19. Pachiadaki MG, Sintes E, Bergauer K, Brown JM, Record NR, Swan BK, et al. Major role of nitrite-oxidizing bacteria in dark ocean carbon fixation. Science. 2017;358:1046-51.

20. Revsbech NP, Larsen LH, Gundersen J, Dalsgaard T, Ulloa O, Thamdrup B. Determination of ultra-low oxygen concentrations in oxygen minimum zones by the STOX sensor. Limnol Oceanogr Methods. 2009;7:371-81.

21. Sun X, Jayakumar A, Ward BB. Community composition of nitrous oxide consuming bacteria in the oxygen minimum zone of the Eastern Tropical South Pacific. Front Microbiol. 2017;8:1-11.

22. Babbin AR, Peters BD, Mordy CW, Widner B, Casciotti KL, Ward BB. Multiple metabolisms constrain the anaerobic nitrite budget in the Eastern Tropical South Pacific. Global Biogeochem Cycles. 2017;31:258-71.

23. Wilke A, Bischof J, Gerlach W, Glass E, Harrison T, Keegan KP, et al. The MG-RAST metagenomics database and portal in 2015. Nucleic Acids Res. 2016;44:D590-4.

24. Nurk S, Meleshko D, Korobeynikov A, Pevzner PA. MetaSPAdes: a new versatile metagenomic assembler. Genome Res. 2017;27:824-34.

25. Li H, Durbin R. Fast and accurate short read alignment with Burrows-Wheeler transform. Bioinformatics. 2009;25:1754-60.

26. Li H, Handsaker B, Wysoker A, Fennell T, Ruan J, Homer N, et al. The sequence alignment/Map format and SAMtools. Bioinformatics. 2009;25:2078-9.

27. Graham ED, Heidelberg JF, Tully BJ. BinSanity: unsupervised clustering of environmental microbial assemblies using coverage and affinity propagation. PeerJ. 2017;5:e3035.

28. Lu YY, Chen T, Fuhrman JA, Sun F, Sahinalp C. COCACOLA: binning metagenomic contigs using sequence COmposition, read CoverAge, CO-alignment and paired-end read LinkAge. Bioinformatics. 2017;33:791-8.

29. Alneberg J, Bjarnason BS, De Bruijn I, Schirmer M, Quick J, Ijaz $\mathrm{UZ}$, et al. Binning metagenomic contigs by coverage and composition. Nat Methods. 2014;11:1144-6.

30. Wu Y-W, Simmons BA, Singer SW. MaxBin 2.0: an automated binning algorithm to recover genomes from multiple metagenomic datasets. Bioinformatics. 2016;32:605-7.

31. Kang DD, Froula J, Egan R, Wang Z. MetaBAT, an efficient tool for accurately reconstructing single genomes from complex microbial communities. PeerJ. 2015;3:e1165.

32. Sieber CMK, Probst AJ, Sharrar A, Thomas BC, Hess M, Tringe $\mathrm{SG}$, et al. Recovery of genomes from metagenomes via a dereplication, aggregation and scoring strategy. Nat Microbiol. 2018;3:836-43.

33. Parks DH, Imelfort M, Skennerton CT, Hugenholtz P, Tyson GW. CheckM: assessing the quality of microbial genomes recovered from isolates, single cells, and metagenomes. Genome Res. 2015;25:1043-55.

34. Tsementzi D, Wu J, Deutsch S, Nath S, Rodriguez-R LM, Burns AS, et al. SAR11 bacteria linked to ocean anoxia and nitrogen loss. Nature. 2016;536:179-83.

35. Glass JB, Kretz CB, Ganesh S, Ranjan P, Seston SL, Buck KN, et al. Meta-omic signatures of microbial metal and nitrogen 
cycling in marine oxygen minimum zones. Front Microbiol. 2015;6:1-13.

36. Stewart FJ, Ulloa O, DeLong EF. Microbial metatranscriptomics in a permanent marine oxygen minimum zone. Environ Microbiol. 2012;14:23-40.

37. Hyatt D, Chen G, Locascio PF, Land ML, Larimer FW, Hauser LJ. Prodigal: prokaryotic gene recognition and translation initiation site identification. BMC Bioinformatics. 2010;11:1-11.

38. Seemann T. Prokka: Rapid prokaryotic genome annotation. Bioinformatics. 2014;30:2068-9.

39. Olm MR, Brown CT, Brooks B, Banfield JF. dRep: A tool for fast and accurate genome de-replication that enables tracking of microbial genotypes and improved genome recovery from metagenomes. ISME J. 2017;11:2864-8.

40. Na SI, Kim YO, Yoon SH, Ha SM, Baek I, Chun J. UBCG: up-todate bacterial core gene set and pipeline for phylogenomic tree reconstruction. J Microbiol. 2018;56:281-5.

41. Stamatakis A. RAxML version 8: a tool for phylogenetic analysis and post-analysis of large phylogenies. Bioinformatics. 2014;30:1312-13.

42. Lee I, Kim YO, Park SC, Chun J. OrthoANI: an improved algorithm and software for calculating average nucleotide identity. Int J Syst Evol Microbiol. 2016;66:1100-3.

43. Eren AM, Esen ÖC, Quince C, Vineis JH, Morrison HG, Sogin ML, et al. Anvi'o: an advanced analysis and visualization platform for 'omics data. PeerJ. 2015;3:e1319.

44. van Dongen S, Abreu-Goodger C. Using MCL to extract clusters from networks. Methods Mol Biol. 2012;804:281-95.

45. Edgar RC. Search and clustering orders of magnitude faster than BLAST. Bioinformatics. 2010;26:2460-1.

46. Edgar RC. MUSCLE: multiple sequence alignment with high accuracy and high throughput. Nucleic Acids Res. 2004;32:1792-7.

47. Letunic I, Bork P. Interactive tree of life (iTOL) v3: an online tool for the display and annotation of phylogenetic and other trees. Nucleic Acids Res. 2016:44:W242-5.

48. Lüke C, Speth DR, Kox MAR, Villanueva L, Jetten MSM. Metagenomic analysis of nitrogen and methane cycling in the Arabian Sea oxygen minimum zone. PeerJ. 2016;4:1-28.

49. Langmead B, Salzberg SL. Fast gapped-read alignment with Bowtie 2. Nat Methods. 2012;9:357-60.

50. Delmont TO, Quince C, Shaiber A, Esen ÖC, Lee ST, Rappé MS, et al. Nitrogen-fixing populations of Planctomycetes and Proteobacteria are abundant in surface ocean metagenomes. Nat Microbiol. 2018;3:804-13.

51. Ngugi DK, Blom J, Stepanauskas R, Stingl U. Diversification and niche adaptations of Nitrospina-like bacteria in the polyextreme interfaces of Red Sea brines. ISME J. 2016;10:1383-99.

52. Thrash JC, Baker BJ, Seitz KW, Temperton B, Campbell LG, Rabalais NN, et al. Metagenomic assembly and prokaryotic metagenome-assembled genome sequences from the Northern Gulf of Mexico "Dead Zone”. Microbiol Res Announc. 2018;7: e01033-18.
53. Konstantinidis KT, Tiedje JM. Prokaryotic taxonomy and phylogeny in the genomic era: advancements and challenges ahead. Curr Opin Microbiol. 2007;10:504-9.

54. Daims H, Lücker S, Wagner M. A new perspective on microbes formerly known as nitrite-oxidizing bacteria. Trends Microbiol. 2016;24:699-712.

55. Anantharaman K, Brown CT, Hug LA, Sharon I, Castelle CJ, Probst AJ, et al. Thousands of microbial genomes shed light on interconnected biogeochemical processes in an aquifer system. Nat Comm. 2016;7:1-11.

56. Pitcher A, Villanueva L, Hopmans EC, Schouten S, Reichart G, Damste JSS. Niche segregation of ammonia-oxidizing archaea and anammox bacteria in the Arabian Sea oxygen minimum zone. ISME J.. 2011;5:1896-904.

57. Füssel J, Lücker S, Yilmaz P, Nowka B, Van Kessel MAHJ, Bourceau P, et al. Adaptability as the key to success for the ubiquitous marine nitrite oxidizer Nitrococcus. Sci Adv. 2017;3:2-11.

58. Han H, Hemp J, Pace LA, Ganesan K, Roh JH, Daldal F, et al. Adaptation of aerobic respiration to low $\mathrm{O}_{2}$ environments. Proc Natl Acad Sci. 2012;109:7947-7947.

59. Lücker S, Wagner M, Maixner F, Pelletier E, Koch H, Vacherie. B, et al. A Nitrospira metagenome illuminates the physiology and evolution of globally important nitrite-oxidizing bacteria. Proc Natl Acad Sci. 2010;107:13479-84.

60. Farrenkopf AM, Luther GW. Iodine chemistry reflects productivity and denitrification in the Arabian Sea: Evidence for flux of dissolved species from sediments of western India into the OMZ. Deep Res Part II Top Stud Oceanogr. 2002;49:2303-18.

61. Griffin BM, Schott J, Schink B. Nitrite, an electron donor for anoxygenic photosynthesis. Science. 2007;316:1870.

62. van de Leemput IA, Veraart AJ, Dakos V, De Klein JJM, Strous M, Scheffer M. Predicting microbial nitrogen pathways from basic principles. Environ Microbiol. 2011;13:1477-87.

63. Koch H, Lücker S, Albertsen M, Kitzinger K, Herbold C, Spieck E, et al. Expanded metabolic versatility of ubiquitous nitriteoxidizing bacteria from the genus Nitrospira. Proc Natl Acad Sci. 2015;112:11371-6.

64. Palatinszky M, Herbold C, Jehmlich N, Pogoda M, Han P, von Bergen $\mathrm{M}$, et al. Cyanate as an energy source for nitrifiers. Nature. 2015;524:105-8.

65. Youngblut MD, Wang O, Barnum TP, Coates JD. Per)chlorate in biology on earth and beyond. Annu Rev Microbiol. 2016;70:435-57.

66. Sandy M, Butler A. Microbial iron acquisition: marine and terrestrial siderophores. Chem Rev. 2009;109:4580-95.

67. Chain P, Lamerdin J, Larimer F, Regala W, Lao V, Land M, et al. Complete genome sequence of the ammonia-oxidizing bacterium and obligate chemolithoautotroph Nitrosomonas europaea. J Bacteriol. 2003;185:2759-73. 